\title{
CrimRxiv
}

\section{A Global History of Execution and the Criminal Corpse}

Richard Ward

Published on: Jul 01, 2015

License: Creative Commons Attribution 4.0 International License (CC-BY 4.0). 
